\title{
GALACTIC COSMIC RAYS-CLOUDS EFFECT AND BIFURCATION MODEL OF EARTH GLOBAL CLIMATE
}

\author{
V.D. Rusov ${ }^{1}$, A.V. Glushkov ${ }^{2}$, V.N. Vaschenko ${ }^{3}$, T.N. Zelentsova ${ }^{1}$, \\ O.T. Mihalys ${ }^{1}$, V.N. Khokhlov ${ }^{2}$, A. Kolos ${ }^{1}$, Zh. Patlashenko \\ ${ }^{1}$ National Polytechnic University, Odessa, Ukraine \\ ${ }^{2}$ Odessa State Environmental University, Odessa, Ukraine \\ ${ }^{3}$ National Antarctic Center, Kiev, Ukraine
}

\begin{abstract}
The possible physical linkage between the cosmic rays - cloud and indirect aerosol effects is discussed using the analysis of the first indirect aerosol effect (Twomey effect) and its experimental representation as the dependence of mean cloud droplet effective radius versus aerosol index defining the column aerosol number.

It is shown that the main kinetic equation of Earth climate energy-balance model is described by the bifurcation equation (relative to the surface temperature of the Earth) in the form of fold catastrophe with two controlling parameters defining the variations of insolation and Earth magnetic field (or cosmic rays intensity in the atmosphere) respectively.

The results of comparative analysis on the time-dependent solution of Earth climate energy-balance model taking into account nontrivial role of galactic cosmic rays and the known experimental data on the paleotemperature from the Vostoc ice core are presented.

In the framework of the bifurcation model (i) the possibility of abrupt glacial climate changes analogous to the Dansgaard-Oeschger events due to stochastic resonance is theoretically argued, (ii) the concept of the climatic sensitivity of water (vapour and liquid) in the atmosphere is introduced. This concept reveals the property of temperature instability in the form of so-called hysteresis loop. On the basis of this concept it is shown that the simulated time series of global ice volume over the past 730 kyr are in good agreement with time series of seawater $\delta^{18} \mathrm{O}$ (ice volume proxy). (iii) Also, the so-called "doubling $\mathrm{CO}_{2}$ " problem is discussed.
\end{abstract}

\section{Introduction}

The fact that galactic cosmic rays (GCR) play one of key parts in the mechanisms responsible for the weather and climate variations observed at our planet is highly plausible [1,2]. Summarizing the outcomes of numerous studies (see, e.g. [1-3]) concerned with the influence of cosmic ray flux (CRF) on atmospheric processes, particularly on the formation of aerosols (condensation centres of water vapour), the following causal sequence of events can be appointed: brighter sun $\rightarrow$ modifications of solar activity and insolation $\rightarrow$ modulation of galactic CRF $\rightarrow$ changes of cloudiness and thunderstorm activity $\rightarrow$ change of albedo $\rightarrow$ variations of weather and climate.

Macroscopic physics accounting for the modulation of CRF via the solar wind is sufficiently evident. The main reason lies in the fact that the coupling between the solar wind and the Earth's magnetosphere is mediated and controlled by the magnetic field in the solar wind through the process of magnetic reconnection [4]. Therefore, the GCR intrusions into the lower atmosphere respond to variations in the Earth's magnetic field induced by its coupling with interplanetary magnetic field and perturbations by eruptive solar events that propagate via the solar wind [5]. As far back as in mid-1950th Forbush [6] provided experimental evidence of rigorous inverse correlation between the cosmic ray intensity and solar activity, and since then many scientists used analogous data on GCR intensity. In particular, Bazilevskaya [7] illustrated the explicit and exact example of inverse correlation between the GCR intensity with energy above $1.5 \mathrm{GeV}$ and the solar activity (protons with energy above $1.0 \mathrm{GeV}$ ) on the basis of the LPI balloon observations during the 1958-2002 years. It should be added that the process of the galactic CRF modulation at the time scales from some days (Forbush phenomenon) to some decades (11-year solar cycle) is experimentally validated with a sufficient clarity, whereas the determination of GCR intensity variations, for example in the past on centennial and millennial timescales, is more intricate problem. The latter is conditioned on the fact that a solution for theory of magnetic reconnection, which is a central problem in magnetospheric physics, is still not extricated in spite of some progress made in the last years [4].

The very important link of GCR intensity and global cloud coverage was found by Svensmark and FriisChristensen [8]. Their results demonstrate the high positive correlation of galactic CRF and cloudiness during long-term cosmic ray modulation in the 11-year solar activity cy cle. Furthermore, it is found that Earth's temperature follows more closely decade variation in galactic CRF and solar cycle length, than other solar activity parameters [9]. Basing on these discoveries, Marsh and Svensmark [1] made a strong assertion "...that solar variability may be linked to climate variability through a chain involving the solar wind, GCR and climate". Moreover, the fact that the influence of solar variability is strongest just in low clouds was experimentally detected. This in turn allows directly pointing out the microphysical mechanism responsible for such an influence and involving aerosol formation, which is enhanced by ionization due to cosmic rays [1].

It is known that aerosols play key part in the cloud formation and directly impact the radiative balance of Earth through a net increase of its albedo. In spite of the fact that their exact role is currently uncertain, in the first place such an effect is caused by a handful of "laws", which were revealed within the confines of atmospheric physics, so-called indirect effects of aerosol on clouds. For example, aerosols can also act as cloud condensation nuclei (CCN), increasing the number of droplets in clouds, which tends to decrease the mean droplet size and may increase the cloud albedo [10], 
depending on the aerosol absorption and cloud optical thickness [11]. This process, referred to as the "Twomey effect" or the "first indirect" aerosol radiative forcing, has a net cooling effect on climate" [12]. From the other hand, the high concentration of aerosol supplies new CCN to condense the excess water vapor as the cloud cools down. Moreover, the smaller droplets are less likely collides with each other and form precipitation. This change in "precipitation efficiency", which is predetermined by the increase in the cloud liquid water content, cloud lifetime and area of coverage, has been termed the second indirect aerosol effect. The global importance of this effect is still not clear [13].

It is obvious that cosmic ray effect and the indirect effect of aerosols on cloud are the similar in that both are driven by change in aerosol number [14]. Therefore, in spite of a number of important distinctions denoted by Carslow et al. [14], it can be supposed with confidence that both these effects are related to each other by drastic and subtle microphysics. The relation becomes apparent at the level of various, but possibly competing, mechanisms of aerosol nucleation and mechanisms of aerosol growth (Fig. 1 from [15]). In this regard, until theory of atmospheric aerosol nucleation can be fully understood, a possible empirical relation between the variations of cosmic ray intensity and the well-known experimental representation of so-called "aerosol index" (AI) can not be fully argued. The aerosol index defines the column aerosol number and can be measured by way of function (Fig. 2) of mean cloud droplet effective radius (hereafter referred to as $\mathrm{CDR}$ ), i.e. by way of $\mathrm{CDR}=\mathrm{f}(\mathrm{AI})[12,13]$.

At the same time, note that the opinions of researchers are various when an accordance of the modulation GCR in the above mentioned causal sequence with the description of weather (stochastic by nature) only or with the deterministic global climate is considered. In our opinion, an answer to this question lies in the known North Atlantic air temperature variations given by Kutzbach and Bryson [16]. The energy spectrum of variation periods shows (Fig. 3) that on the right of "window", i.e. on the right of profound and broad minimum, there is a spectrum of

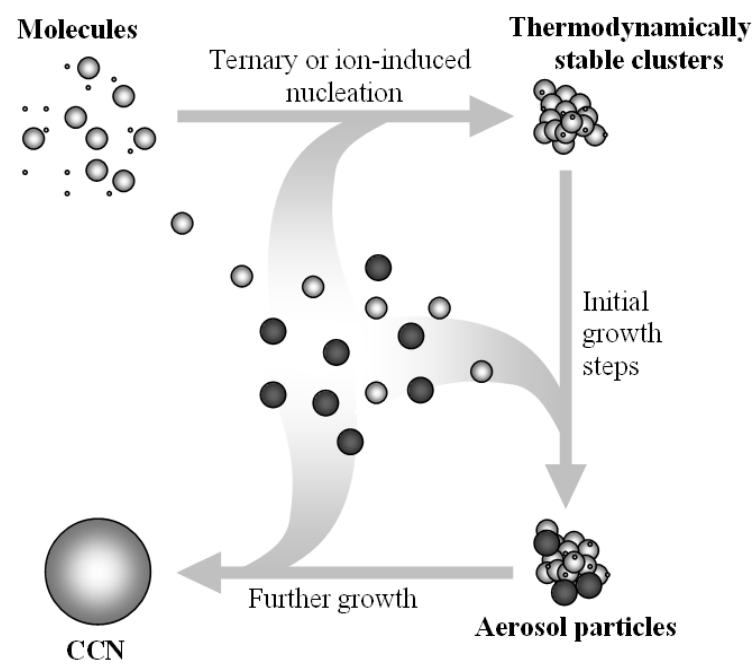

Fig. 1. How particles form and grow [Kulmala, 2003]: Nucleation may involve homogeneous ternary watersulfuric acid-ammonia mixture or may be ion-induced. The initial steps of growth include activation of inorganic clusters by soluble organic molecules, heterogeneous nucleation of insoluble organic vapors on inorganic clusters, and chemical reactions of organic molecules at surfaces of inorganic clusters. Finally, cloud condensation nuclei (CCN) form through addition of organic and sulfuric acid molecules.

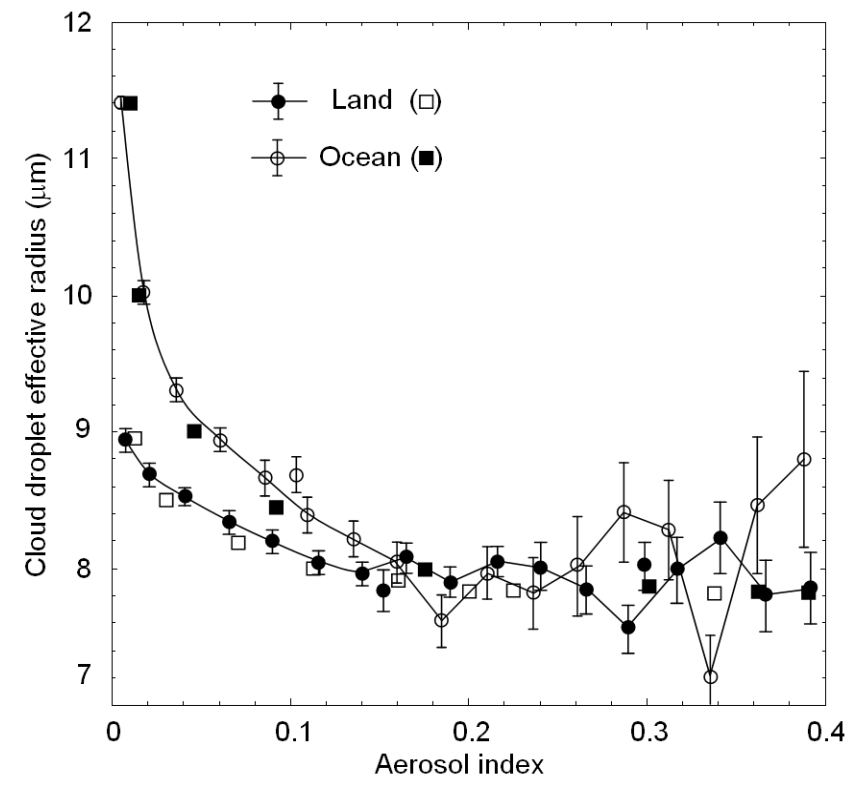

Fig. 2. Effect of aerosol on cloud droplet: mean cloud droplet effective radius (CDR) as a function of aerosol load [Breon et al., 2002]. The two curves show the mean CDR as a function of aerosol index (AI) for land (lower curve) and 
ocean (upper curve). The error bars represent the confidence level of the mean value, i.e. $\sigma / \sqrt{n-2}$, where $\mathrm{n}$ and $\sigma$ are the number of CDR measurements within the bin and their standard deviation [Breon et al., 2002]. The values of empirical relation CDR=f (AI) in the form of Eq. (1) for land ( $\mathbf{a})$ and ocean ( $\square$ ) are presented.

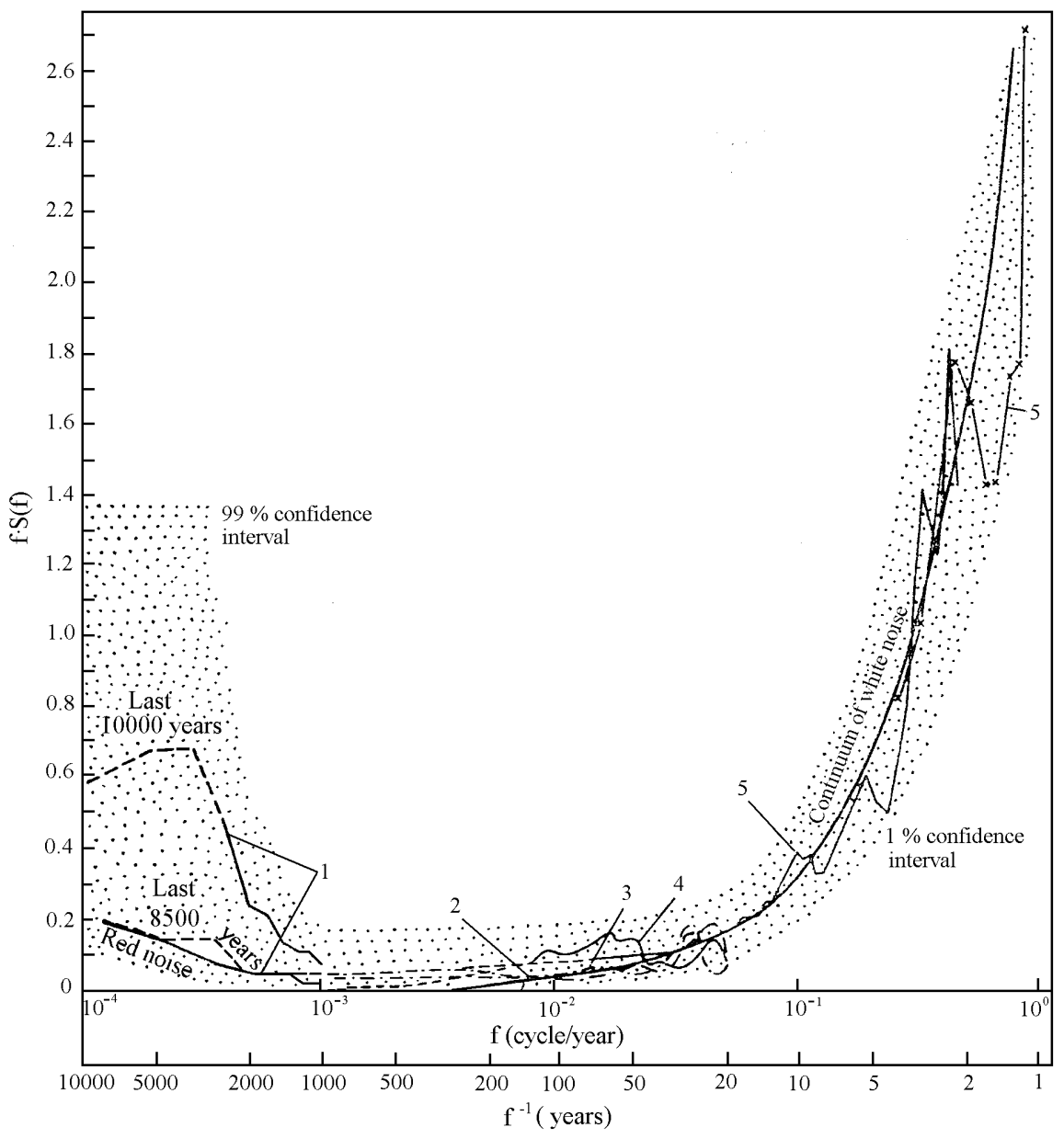

Fig. 3. Combined spectrum of the air temperature variations in the North-Atlantic sector of the terrestrial globe [Kutzbach and Bryson, 1974]: $f$ are frequencies, cycle/year; S(f) is spectral density; 1 - Central England, paleobotany; 2 - the same, chronicles; 3 - Iceland, chronicles; 4 - Greenland, obtained by $\delta^{18} \mathrm{O} ; 5$ - Central England, by Manley series [Kutzbach and Bryson, 1974].

weather variations determined by the white noise, whereas a spectrum of long-period climate fluctuations determined by the red noise lies on the left of this minimum. Note that the red noise possesses some predictability (determinacy) in contrast to the white noise. Also, an intermediate spectrum of stochastic climate with the 1/f noise corresponds to the profound and broad minimum.

Thus the spectrum of temperature variations within the Earth climate system (ECS) shows that the averaging of ECS parameters at appropriate time intervals is necessary to describe the weather and climate adequately. It is also obvious that the averaging periods for ECS parameters in the case of stochastic description of weather and "intermediate" climate must be over the range 0-30 and 30-1000 years, respectively, whereas the deterministic description of global climate evolution necessitates averaging periods $\geq 1000$ years.

It is surely ascertained [17] that the spectral density of virtual axial dipole moment (VADM) fluctuations in the magnetic field of the Earth displays the variations with a period $\sim 100 \mathrm{kyr}$, which correspond to the variations in the orbital eccentricity of the Earth, in the spectrum of paleointensity periodicities during the past 2.25 million years. Moreover, Yamasaki and Oda [17] suggested that the geomagnetic field is, in some way, modulated by orbital eccentricity variations. In our opinion, this effect can be phenomenologically explained if suppose that gravitational field of the Sun (by way of indirect influence on the angular velocity of the Earth) affects convection processes (hydromagnetic dynamo) in the terrestrial liquid core and, thereby, "delegates" the variations of intensity with periods of $\sim 100 \mathrm{kyr}$ to the terrestrial magnetic field. This mechanism for the precession of the Earth as the cause of geomagnetism was for the first time proposed by Malkus [18]. It is interestingly to note that such a mechanism was used by Consolini and De Michelis [19] to explain a possible link between GCR and periodic changes of Earth's orbital parameters on basis of stochastic resonance in geomagnetic polarity reversals.

Thus, in spite of limited lucidity of reason responsible to the appearance of eccentricity-driven frequencies in the magnetic-field paleointensity spectrum, the conclusion can be made that millennial fluctuations of Earth's magneticfield intensity are peculiar modulator of GCR at time scales $\geq 1000$ years. It is also obvious that such a type of modulation for the GCR relates directly to "deterministic" climate at the millennial time scale, and this fact can be used as a driving parameter in models of global climate. 
This paper have for an object a development of energy-balance model of climatic response to orbital variations, which takes into account an influence of galactic cosmic rays on global climate.

\section{On possible relation between cosmic rays-cloud and indirect aerosol effects}

It is known, that the indirect observation of Twomey effect (the first indirect aerosol effect) can be made by comparing cloud droplet size and aerosol concentration. In fact, a dependence of CDR on AI (Fig. 2) is measured in actual satellite observations by means of radiometers $[12,13]$. This is determined by the fact that "CDR is more sensitive to the aerosol index than to the optical thickness, which is to be expected, because the aerosol index is function of the CCN (cloud condensation nuclei) concentration" [12]. It can be easily shown that the observed dependence of CDR on AI over oceans and land is represented (with approximation sufficient for our purpose) as the empirical dependence

$$
A I=\left[\frac{1}{\left(0.6 r_{e f f}-4.385\right) r_{e f f}}-\frac{\eta}{r_{e f f}}\right]^{1.429}, \quad \eta=\left\{\begin{array}{l}
0, \text { over ocean } \\
0.63, \text { over land }
\end{array}\right.
$$

where $\mathrm{r}_{\mathrm{eff}}=\left\langle\mathrm{r}^{3}\right\rangle /\left\langle\mathrm{r}^{2}\right\rangle$ is the mean $\mathrm{CDR}$, and $\mathrm{r}$ is the radius of the cloud droplets.

The number of aerosol particles that may act as $\mathrm{CCN}, N_{C C N}$, and the number of cloud droplets, $N_{d}$, are approximately related through [20]

$$
N_{d} \approx\left(N_{C C N}\right)^{\alpha} \text {. }
$$

Taking into account that, on the one hand, cloud process models and measurements indicate that $\alpha$ is on the order of 0.7 [12], and, on the other hand, AI $\sim \mathrm{N}_{\mathrm{CCN}}$ [12], using Eqs. (1) and (2) we introduce the following expression for the concentration of cloud droplets:

$$
N_{d} \approx \frac{1}{\left(0.6 r_{e f f}-4.385\right) r_{e f f}}-\frac{\eta}{r_{e f f}} \text {. }
$$

Then the volume of liquid water in the atmosphere "over the ocean" or "over the land", $V_{W}$, equals to

$$
V_{W}=p_{i} V_{a t m} \cdot \frac{4}{3} \pi\langle r\rangle^{3} N_{d}=p_{i} V_{a t m} \cdot \frac{4 \pi}{3} \frac{r_{e f f}^{2}}{k_{r}^{3}}\left(\frac{1}{0.6 r_{e f f}-4.385}-\eta\right) \text {, }
$$

where $\mathrm{V}_{\text {atm }} \cong$ const is the total volume of the atmosphere, $\mathrm{p}_{\mathrm{i}}$ is the portion of the atmosphere volume "over the ocean" or "over the land", $\langle\mathrm{r}\rangle \approx \mathrm{k}_{\mathrm{r}} \cdot \mathrm{r}_{\text {eff }}$ is the mean radius of cloud droplets. Therefore, the averaged total volume of liquid water in the atmosphere can be defined as

$$
\begin{aligned}
& \left\langle V_{W}\right\rangle=V_{\text {atm }} \cdot \frac{4 \pi}{3} \frac{r_{\text {eff }}^{2}}{k_{r}^{3}}\left[\frac{1}{0.6 r_{\text {eff }}-4.385}-\frac{S_{\text {land }} \Lambda}{S_{\text {ocean }}+S_{\text {land }}} 0.63\right], \\
& \Lambda=\Lambda\left(r_{\text {eff }}-7.7\right)-\Lambda\left(r_{\text {eff }}-9.6\right),
\end{aligned}
$$

where $\Lambda(\mathrm{x})$ is the integral of $\delta$-function

$$
\Lambda(x)=\int_{-\infty}^{x} \delta(z) d z= \begin{cases}1, & x \geq 0 \\ 0, & x<0\end{cases}
$$

$\mathrm{S}_{\text {land }}$ and $\mathrm{S}_{\text {ocean }}$ are the areas of land and oceans respectively; $\mathrm{S}_{\text {land }} /\left(\mathrm{S}_{\text {land }}+\mathrm{S}_{\text {ocean }}\right) \cong 0.29$.

It is noteworthy that the pronounced minimum at $r_{\text {eff }} \approx 14 \mu \mathrm{k}$ appears in the solution of Eq. (5) (see Fig. 4). This minimum can be seemingly associated with the so-called precipitation threshold [21]. In further considerations, we take into account the left-hand portion (with respect to the minimum) only of this relation. 


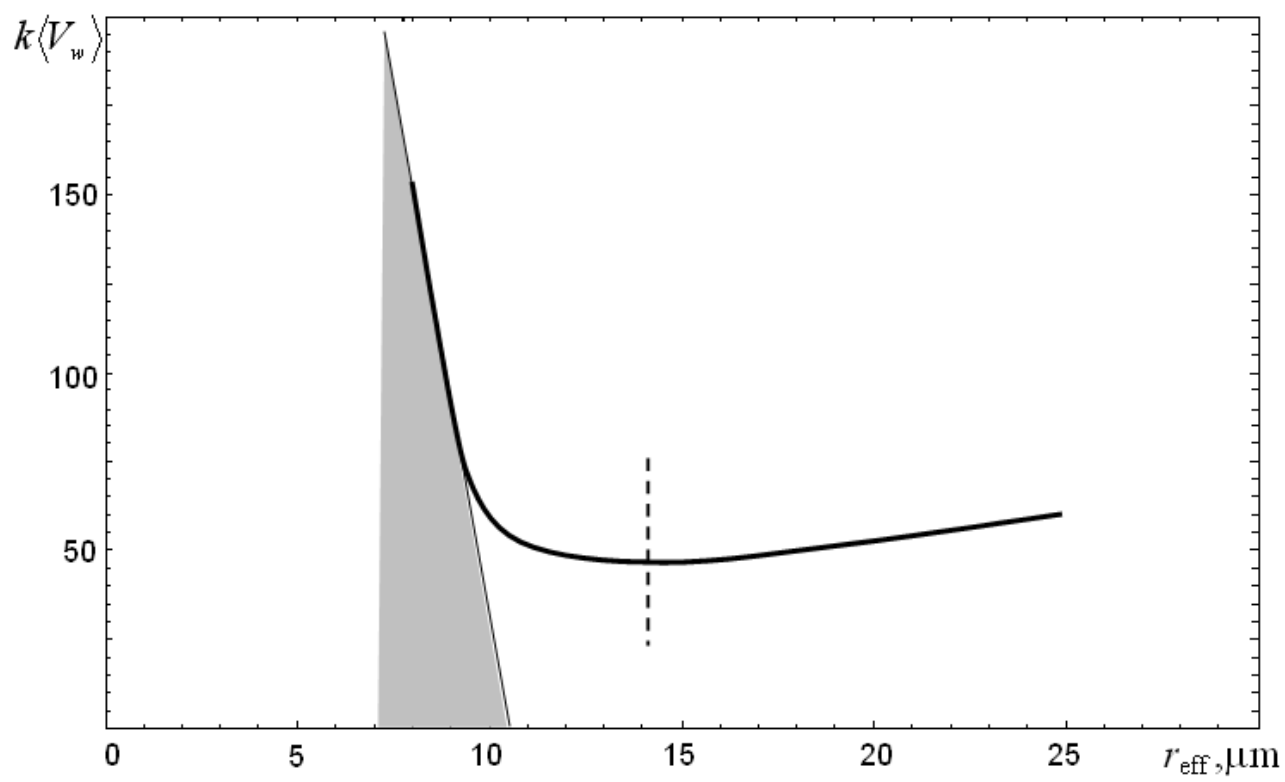

Fig. 4. Mean total volume of liquid water $\left\langle\mathrm{V}_{\mathrm{w}}\right\rangle$ in the atmosphere as a function of cloud droplet effective radius (CDR). Minimum at $\mathrm{r}_{\mathrm{eff}} \approx 14 \mu \mathrm{k}$ (vertical dotted line) corresponds to the so-called precipitation threshold. Left-hand part with respect to the minimum (hatched) is defined by the inverse linear dependence $\left\langle\mathrm{V}_{\mathrm{w}}\right\rangle$ from $\mathrm{CDR}$.

Eq. (5) can be reduced since the actual variations of temperature, $\Delta \mathrm{T}$, which are assigned to the "warm" and "ice" ages of the Earth climate, lie in the relatively narrow range. Note that the temperature fluctuations derived from the Vostoc ice core [22] are $\pm 4 \div 6 \mathrm{~K}$. Taking into account this fact, it is easy to show on the basis of experimental data $[21,23]$ that due to inverse linear dependence the small increments of the average CDR correspond to such increments $\Delta \mathrm{T}$, i.e., $\Delta \mathrm{r}_{\text {eff }} \approx 2 \div 3 \mu \mathrm{k}$. Therefore, it can be supposed that the actual scenarios of global climate "know" and "feel" the relatively small range of the CDR values from a quantity of permitted values $\left(\mathrm{r}_{\mathrm{eff}} \approx 8 \div 14 \mu \mathrm{k}\right)$ lying in the left of the precipitation threshold line in Fig. 4. This property of climatic scenarios allows, in turn, reducing the expression for the averaged total volume of liquid water in the atmosphere. Then, a first approximation of Eq. (5) can be written as an inverse linear dependence on the CDR (see Fig. 4) or as a direct one from the temperature

$$
\left\langle V_{w}\right\rangle \approx k_{w}\left(a-b r_{e f f}\right)=a_{w}+b_{w} T \text {. }
$$

Now, the following procedure to calculate the total water (vapour and liquid) in the atmosphere, $\left\langle\mathrm{V}_{\mathrm{w}+\mathrm{v}}\right\rangle$ can be offered. There is a general argument to suppose that the theoretical dependence of water vapour volume $\left\langle\mathrm{V}_{\mathrm{v}}\right\rangle$ on the temperature is the same as Eq. (6), but this dependence differs in quantitative characteristics only. Investigating the bistabillity of $\mathrm{CCN}$ concentrations and thermodynamics in the cloud-topped boundary layer (CTPL), Baker and Charlson [24] showed that the total water (vapour and liquid) and the temperature of CTPL are directly proportional to the sea surface temperature. Also, Albrecht [25] showed that there is a linear dependence of cloud fraction on the sea surface temperature. This allows supposing that the total volume of water (vapour and liquid) in the atmosphere $\left\langle\mathrm{V}_{\mathrm{w}+\mathrm{v}}\right\rangle$ is directly proportional to surface temperature

$$
\left\langle V_{w+v}\right\rangle=\left\langle V_{w}\right\rangle+\left\langle V_{v}\right\rangle \sim T
$$

Therefore, if Eq. (6) is linear with regard to the temperature, in view of Eq. (7) the dependence for the total volume of water vapour $\left\langle V_{v}\right\rangle$ must also be linear with regard to the temperature. Then, Eq. (7) can be rewritten as follows

$$
\left\langle V_{w+v}\right\rangle \approx a_{w v}+b_{w v} T
$$

As it was mentioned earlier, the cosmic ray effect and the indirect effect of aerosols on cloud are the similar in that both are driven by change in aerosol number [14]. Although these effects, in our opinion, are connected by a common "microphysics", Eq. (1) not contains a term responsible for the cosmic ray effect. In further paragraphs, we try to examine reasons why such a term is absent in Eq. (1) and to "rehabilitate" it in the general case of various time intervals.

It is known [12] that the dependence (1) shown in Fig. 2 was experimentally derived for the relatively short period (March-May 1997). This means that Eq. (1) describes a response of atmospheric microprocesses to an impact of slightly-varying insolation under the settled intensity of GCR. If experiments were more durational, i.e. were run, for example, during long-term cosmic ray modulation in the 11-year solar activity cycle, the additional influence of GCR intensity variations (hereafter, $\Delta \Phi_{\mathrm{GCR}}$ ) on the AI distribution should also be apparent.

On the one hand, such an influence must be conditioned on the effect of inverse correlation between the magnitude of GCR intensity (hereafter, $\Phi$ ) and land temperature, T, which was experimentally ascertained by Svensmark [9]. This fact can be conditionally written as follows 


$$
\Phi \downarrow \uparrow \Rightarrow T \uparrow \downarrow,
$$

where the arrows " $\uparrow "$ and " $\downarrow$ " show the increase and decrease of physical quantity respectively.

On the other hand, it can be revealed on basis of known experiments [21,23]] relating to the measurements of $\mathrm{T}_{\text {cloud }}-\mathrm{r}_{\text {eff }}$ (hereafter, $\mathrm{T}_{\text {cloud }}$ is the cloud temperature) relations by the method of Rosenfeld and Lensky [23] that in the case of spatio-temporal averaging of $T_{\text {cloud }}-r_{\text {eff }}$ relation measurements, which was carried out over the oceans, the dependence between these parameters, $\mathrm{T}_{\text {cloud }}$ and $\mathrm{r}_{\text {eff }}$, is practically linear:

$$
r_{\text {eff }}=a_{r}-b_{r} T_{\text {cloud }} \text {, }
$$

Hereinafter, taking into account the Eddington approximation for the outer boundary of atmosphere, we suppose that $\mathrm{T}_{\text {cloud }} \sim$ const $\cdot \mathrm{T}$. Then, subject to Eqs. (10) and (1), the following conditional dependence can be written

$$
T \uparrow \downarrow \Rightarrow r_{\text {eff }} \downarrow \uparrow \Rightarrow A I \uparrow \downarrow .
$$

At last, by virtue of dependences (7)-(9)-(10) we can suppose that it is possible to take into account the additional influence of GCR intensity variations $\Delta \Phi_{\mathrm{GCR}}$ in the atmosphere on aerosol index distribution as the additional variation of total volume $\left\langle\mathrm{V}_{\mathrm{w}+\mathrm{v}}\right\rangle$, which was obtained by Eq.(1):

$$
\left\langle V_{w+v}\right\rangle \approx\left(a_{w v}+b_{w v} T\right)\left(1+\frac{M-M_{0}}{M_{0}}\right)=\left(a_{w v}+b_{w v} T\right) \frac{M}{M_{0}}=\left(a_{w v}+b_{w v} T\right) M_{\oplus}^{*}
$$

where following approximate equation was used:

$$
\frac{M-M_{0}}{M_{0}} \approx-\frac{\Phi-\Phi_{0}}{\Phi_{0}},
$$

and $M_{\oplus}^{*}$ is geomagnetic paleointensity, which measured for the past 2.25 million years [17], $\Phi_{0}$ and $\mathrm{M}_{0}$ are the cosmic rays flux and Earth's magnetic field measured, for example, in October 1965 [9]. Note that physical meaning of dependence (12) corresponds to the main hypothesis of new model for the glacial cycles. This model is resulted from the analysis of high-precision experimental paleorecords. The model lies in the fact that the forcing mechanisms "are initially driven not by the insolation cycles, but by cosmic ray changes, probably through their effect on clouds" [26].

It is noteworthy that we do not consider the above arguments as any substantiation of Eq. (12). It is obvious that an experiment is only possible way to validate theoretic assumptions. Since the direct experimental validation for Eq. (12) is now inaccessible to us, we propose the following scheme to verify our assumptions.

By means of Eq. (12), the total volumes of condenced water in the atmosphere, $\mathrm{V}_{\mathrm{w}}$, and water vapour, $\mathrm{V}_{\mathrm{v}}$, are calculated. These calculations allow estimating the masses of $V_{w}$ and $V_{v}$ and, consequently, the re-emission energies causing the total radiant energy of water, $\Delta \mathrm{E}_{\mathrm{w}}$, and water vapour, $\Delta \mathrm{E}_{\mathrm{v}}$, subject to the temperature of ECS. Then, if an application of these terms in the energy-balance model of Earth's climate results in the solution of equation concerning the temperature of ECS, which is well-consistent with experimental data on the paleotemperature (e.g., the Vostoc ice core data [22] over the past $420 \mathrm{kyr}$ ), our assumptions can be considered as acceptable. In our opinion, experimental investigations on the possible synergetic (with respect to insolation action) influence of GCR intensity on to the aerosol index distribution, $\mathrm{AI}=\mathrm{f}(\mathrm{CDR})$, can refute or confirm our assumptions.

Such a view of Eq. (12) is the direct consequence of basic assumption of our model: we assume that the temperature of ECS is defined not single controlling parameter, but two ones - the insolation variations and GCR intensity fluctuations (or fluctuations of Earth's magnetic field). In the next section, we consider the energy-balance model of Earth's climate with two controlling parameters.

\section{Catastrophe theory and energy-balance model of global climate}

By act of energy conservation law, the actual heat rate of Earth's radiation is approximately equal to the difference between the rate of long-wave radiation of Earth's surface, $\mathrm{I}(\mathrm{T}, \mathrm{t})$, and the heat energy re-emitted by the liquid water, $\mathrm{G}_{\mathrm{w}}(\mathrm{T}, \mathrm{t})$, water vapour, $\mathrm{G}_{\mathrm{v}}(\mathrm{T}, \mathrm{t})$, and carbon dioxide, $G_{\mathrm{CO}_{2}}(\mathrm{~T}, \mathrm{t})$. With the purpose of simplification, we not consider other greenhouse gases. Since the radiant equilibrium can be achieved at time scales of $10^{4}-10^{5}$ years, the inclusion of greenhouse effect results in the following energy-balance equations for the ECS (see also Fig. 5)

$$
U(T, t)=P_{\text {Sun }}(t) \cdot[1-\alpha(T)]-I_{E a r t h}(T)+\frac{1}{2} G_{w}(T, t)+\frac{1}{2} G_{v}(T, t)+\frac{1}{2} G_{C O_{2}}(T, t),
$$

where the first member of equations $\mathrm{U}(\mathrm{T}, \mathrm{t})$, if it not equals to zero, describes a magnitude of so-called "inertial" rate of heat variations in the ECS; $\mathrm{P}_{\text {Sun }}(\mathrm{t})=(1 / 4) \mathrm{S}(\mathrm{t}) \cdot \gamma$ is the heat flow of solar radiation at the top of atmosphere $(\mathrm{W}) ; \mathrm{S}(\mathrm{t})$ is the 
insolation $\left(\mathrm{W} / \mathrm{m}^{2}\right) ; \alpha$ is the albedo of $\mathrm{ECS}$; $\mathrm{I}_{\mathrm{Earth}}=\gamma \delta\left(\sigma \mathrm{T}^{4}\right), \mathrm{W} ; \delta=0.95$ is the Stephen-Boltzmann constant; T is the temperature of Earth's surface $(\mathrm{K}) ; \gamma$ is the area of atmosphere outer boundary $\left(\mathrm{m}^{2}\right)$; $\mathrm{t}$ is the time, for which the energy balance is considered.

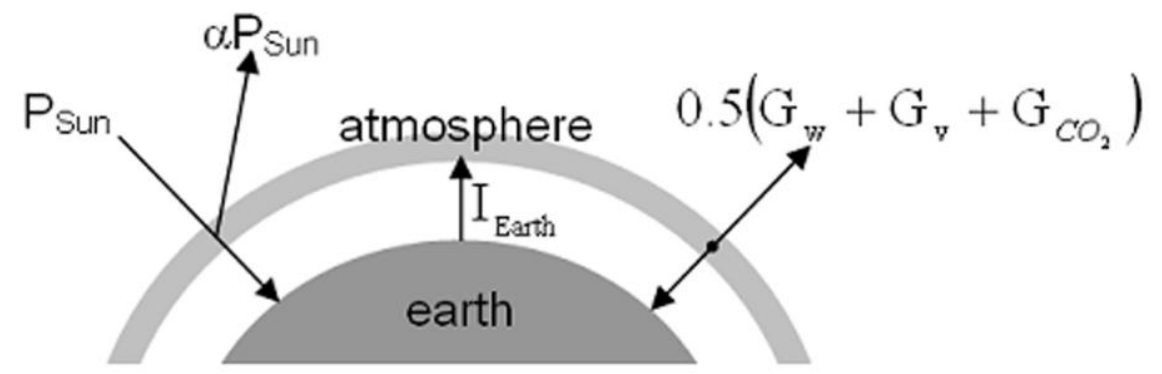

Fig. 5. Balance of the absorbed and emitted energy currents on the surface of the Earth.

First, examine the question on functional dependence for the rate of heat energy $G_{w}(T, t)$ re-emitted by the liquid water on the temperature. It is obvious that Eq. (10) for mean total volume of liquid water in the atmosphere allows writing down the following relation for the rate of re-emission

$$
G_{w}(T, t)=\varepsilon_{w} \rho_{w}\left\langle V_{w}\right\rangle
$$

where $\varepsilon_{\mathrm{w}}$ is the mean radiant power for the unit mass of liquid water, $\rho_{\mathrm{w}}$ is the density of liquid water.

It is possible to suppose that the average emissive power of liquid water unit mass $\varepsilon_{\mathrm{w}}$ as a first approximation depends on the ECS temperature. Then taking into account Eq. (11) let us introduce the linear dependence of $\varepsilon_{\mathrm{w}}$ on the ECS temperature in Eq. (15):

$$
G_{w}(T, t)=\frac{\gamma H}{\left\langle V_{a t m}\right\rangle} \varepsilon_{w} \rho_{w}\left\langle V_{w}\right\rangle=\frac{\gamma H}{\left\langle V_{a t m}\right\rangle} \rho_{w}\left(a_{w \varepsilon} T^{2}+b_{w \varepsilon} T+c_{w \varepsilon}\right) M_{\oplus}^{*} .
$$

In a similar manner it is possible to obtain the expression of the thermal power of energy $G_{v}(T, t)$ re-emitted by vapour water:

$$
G_{v}(T, t)=\frac{\gamma H}{\left\langle V_{a t m}\right\rangle} \varepsilon_{v} \rho_{v}\left\langle V_{v}\right\rangle=\frac{\gamma H}{\left\langle V_{a t m}\right\rangle} \rho_{v}\left(a_{v \varepsilon} T^{2}+b_{v \varepsilon} T+c_{v \varepsilon}\right) M_{\oplus}^{*},
$$

где $\varepsilon_{\mathrm{v}}$ is the average emissive power of vapour water unit mass, $\mathrm{W} / \mathrm{kg} ; \rho_{\mathrm{v}}$ is the vapour water density, $\left\langle\mathrm{V}_{\mathrm{atm}}\right\rangle \approx \gamma \mathrm{H}$.

To examine a question on the functional dependence for the rate of heat energy $G_{\mathrm{CO}_{2}}(\mathrm{~T}, \mathrm{t})$ on the temperature of ECS, use the analysis of known experimental data on the variations of temperature and carbon dioxide content over the past $420 \mathrm{kyr}$ from the Vostoc ice core [22]. It is obvious that these data are highly linear correlated. Therefore, it can be supposed that the dependence for the rate of heat energy on the temperature of ECS is also linear

$$
G_{\mathrm{CO}_{2}}(T, t) \approx \frac{\gamma \mathrm{H}}{\left\langle V_{\mathrm{atm}}\right\rangle}\left(a_{\mathrm{CO}_{2}} T+b_{\mathrm{CO}_{2}}\right) .
$$

It must be added that the dependence for the effective value of albedo on the temperature of ECS is chosen as the continuous parameterization

$$
\alpha=\alpha_{0}-\eta_{\alpha} \cdot(T-273) \text {. }
$$

Eq. (19) represents well, for example, the albedo behaviour (under $\alpha_{0}=0.7012, \eta_{\alpha}=$ $0.0295 \mathrm{~K}^{-1}$ ) in the temperature range of 282-290 K.

Finally, assembling all partial contributions of heat fluxes (16)-(18) and $\mathrm{I}_{\text {Earth }}=\gamma \delta\left(\sigma \mathrm{T}^{4}\right)$ into the final energybalance expression (14), we derive

$$
U^{*}(T, t)=\frac{1}{4} T^{4}+\frac{1}{2} a(t) \cdot T^{2}+b(t) \cdot T,
$$

where

$$
\begin{aligned}
& a(t)=-\frac{1}{4 \delta \sigma} a_{\mu} M_{\oplus}^{*}(t), \\
& b(t)=-\frac{\eta_{\alpha}}{32 \delta \sigma}\left[S(t)+\frac{4}{\eta_{\alpha}} \beta+\frac{4}{\eta_{\alpha}} b_{\mu} M_{\oplus}^{*}(t)\right],
\end{aligned}
$$




$$
\begin{aligned}
& U^{*}(T, t)=\frac{1}{4 \gamma \delta \sigma}\left[\frac{1}{4}\left(1-\alpha_{\alpha}-273 \eta_{\alpha}\right) S(t)+\frac{1}{2} b_{\mathrm{CO}_{2}} H /\left\langle V_{a t m}\right\rangle+\frac{1}{2} c_{\mu} M_{\oplus}^{*}-U(T, t)\right], \\
& \beta=a_{\mathrm{CO}_{2}} H /\left\langle V_{a t m}\right\rangle, \quad\left[W / m^{2} K\right] \\
& a_{\mu}=\left(\rho_{w} a_{w \varepsilon}+\rho_{v} a_{v \varepsilon}\right) H /\langle a t m\rangle, \quad\left[W / m^{2} K^{2}\right] \text {, } \\
& b_{\mu}=\left(\rho_{w} b_{w \varepsilon}+\rho_{v} b_{v \varepsilon}\right) H /\left\langle V_{a t m}\right\rangle, \quad\left[W / m^{2} K\right] \text {, } \\
& \left.c_{\mu}=\left(\rho_{w} c_{w \varepsilon}+\rho_{v} c_{v \varepsilon}\right) H /\left\langle V_{a t m}\right\rangle, \mid W / m^{2}\right\rfloor \text {, }
\end{aligned}
$$

here $\mathrm{a}_{\mathrm{w} \varepsilon}, \mathrm{a}_{\mathrm{v} \varepsilon}, \mathrm{b}_{\mathrm{w} \varepsilon}, \mathrm{b}_{\mathrm{v} \varepsilon}, \mathrm{c}_{\mathrm{w} \varepsilon}, \mathrm{c}_{\mathrm{v} \varepsilon}$ and $a_{\mathrm{CO}_{2}}$ are constant coefficients, whose dimensions determined by Eqs. (16), (17) and (18) respectively.

It is obvious that Eq. (20) describes the collection of energy-balance functions $\Delta U(T, a, b)$, which depend on two controlling parameters, $\mathrm{a}(\mathrm{t})$ and $\mathrm{b}(\mathrm{t})$. Also, this collection represents so-called potential of fold catastrophe [27].

In future, we will be interested by an equation of fold catastrophe (20) relative to increment $\Delta \mathrm{T}=\mathrm{T}-\mathrm{T}_{0}$ of the following view: $U\left(T_{0}+\Delta T, a, b\right)-U\left(T_{0}, a, b\right)=\Delta U$, where $T_{0}$ is the mean temperature of ECS averaged at the respective time interval $\Delta \mathrm{t}$. Also, the increment for the first right-hand term of Eq. (20) can be represent in the following equivalent form

$$
\left(T_{0}+\Delta T\right)^{4}-T_{0}^{4} \cong 7 \cdot 10^{-3} \cdot T_{0}^{3} \cdot(\Delta T)^{4}+4 \cdot T_{0}^{3} \cdot \Delta T, \quad \text { for } \quad \Delta T=0 \div 4 K,
$$

for which the mean error of approximation at given range of temperature not exceeds $0.01 \%$.

Let us remind that the normalized variations of insolation, $\Delta \hat{S}=\left(S-S_{0}\right) / \sigma_{S}$, with mean value $<\Delta \hat{S}>=0$ and dispersion $\sigma_{\Delta \hat{S}}^{2}=1$ is applied more often for the simulation of the ECS.

Deriving an equation in the form of Eq. (20) with respect to $\Delta \mathrm{T}$, the following expression for the increment of heat rate $\Delta \mathrm{U}^{*}$ can be defined

$$
\Delta U^{*}(\Delta T, t)=\frac{1}{4} \Delta T^{4}+\frac{1}{2} \tilde{a}(t) \cdot \Delta T^{2}+\tilde{b}(t) \cdot \Delta T
$$

where

$$
\begin{aligned}
& \tilde{a}(t)=-\frac{37.6}{\sigma T_{0}^{3}} a_{\mu} M_{\oplus}^{*}(t)=-\tilde{a}_{0} \cdot M_{\oplus}^{*}(t) \\
& \tilde{b}(t)=-\frac{4.7 \eta_{\alpha} \sigma_{S}}{\sigma T_{0}^{3}}\left[\Delta \hat{S}(t)+\frac{1}{\sigma_{S}}\left(S_{0}-\frac{32 \delta \sigma T_{0}^{3}}{\eta_{\alpha}}\right)+\frac{4}{\eta_{\alpha}} \cdot \frac{\beta}{\sigma_{S}}+\frac{4}{\eta_{\alpha}} \cdot \frac{2 a_{\mu} T_{0}+b_{\mu}}{\sigma_{S}} M_{\oplus}^{*}(t)\right]= \\
& =-\frac{4.7 \eta_{\alpha} \sigma_{S}}{\sigma T_{0}^{3}}\left[\Delta \hat{S}(t)+\frac{4}{\eta_{\alpha}} \cdot \frac{\beta}{\sigma_{S}}+\frac{4}{\eta_{\alpha}} \cdot \frac{2 a_{\mu} T_{0}+b_{\mu}}{\sigma_{S}} M_{\oplus}^{*}(t)\right]= \\
& =-\tilde{b}\left[\Delta \hat{S}(t)+\frac{4}{\eta_{\alpha}} \cdot \Delta \hat{\mathfrak{R}}_{C O_{2}}+\frac{4}{\eta_{\alpha}} \cdot \Delta \hat{\mathfrak{R}}_{w+v} M_{\oplus}^{*}(t)\right]
\end{aligned}
$$

It is obvious that the preservation of invariance for the internal operational structure, which exists during the transition from Eq. (20) to Eq. (24), results simultaneously in the preservation of identical internal operational structure of Eq. (21) and (24) as well as Eq. (22) and (26) for the respective pairs of controlling parameters a(t), $\tilde{a}(\mathrm{t})$ and $\mathrm{b}(\mathrm{t}), \tilde{b}(\mathrm{t})$. If this condition is met for the pair of controlling parameters $\mathrm{a}(\mathrm{t}), \tilde{a}(\mathrm{t})$, then in order to this condition would met for the pair $\mathrm{b}(\mathrm{t}), \tilde{b}(\mathrm{t})$ the internal structure of Eq. (26) must be complied with the following physical requirement

$$
\frac{1}{\sigma_{S}}\left(S_{0}-\frac{32 \delta \sigma T_{0}^{3}}{\eta_{\alpha}}\right)=0 \cdot
$$

A validity of above remark can be easily ascertained by the test of Eq. (27) under the values of insolation $\mathrm{S}_{0}=1375.5 \mathrm{~W} / \mathrm{m}^{2}$, global temperature $\mathrm{T}_{0}=286.6 \mathrm{~K}$, and $\eta_{\alpha}=0.0295$.

The canonical form of the variety of the fold catastrophe, which represents a set of points $(\Delta T, \tilde{a}, \tilde{b})$, satisfies the equation (see Fig. 6a): 

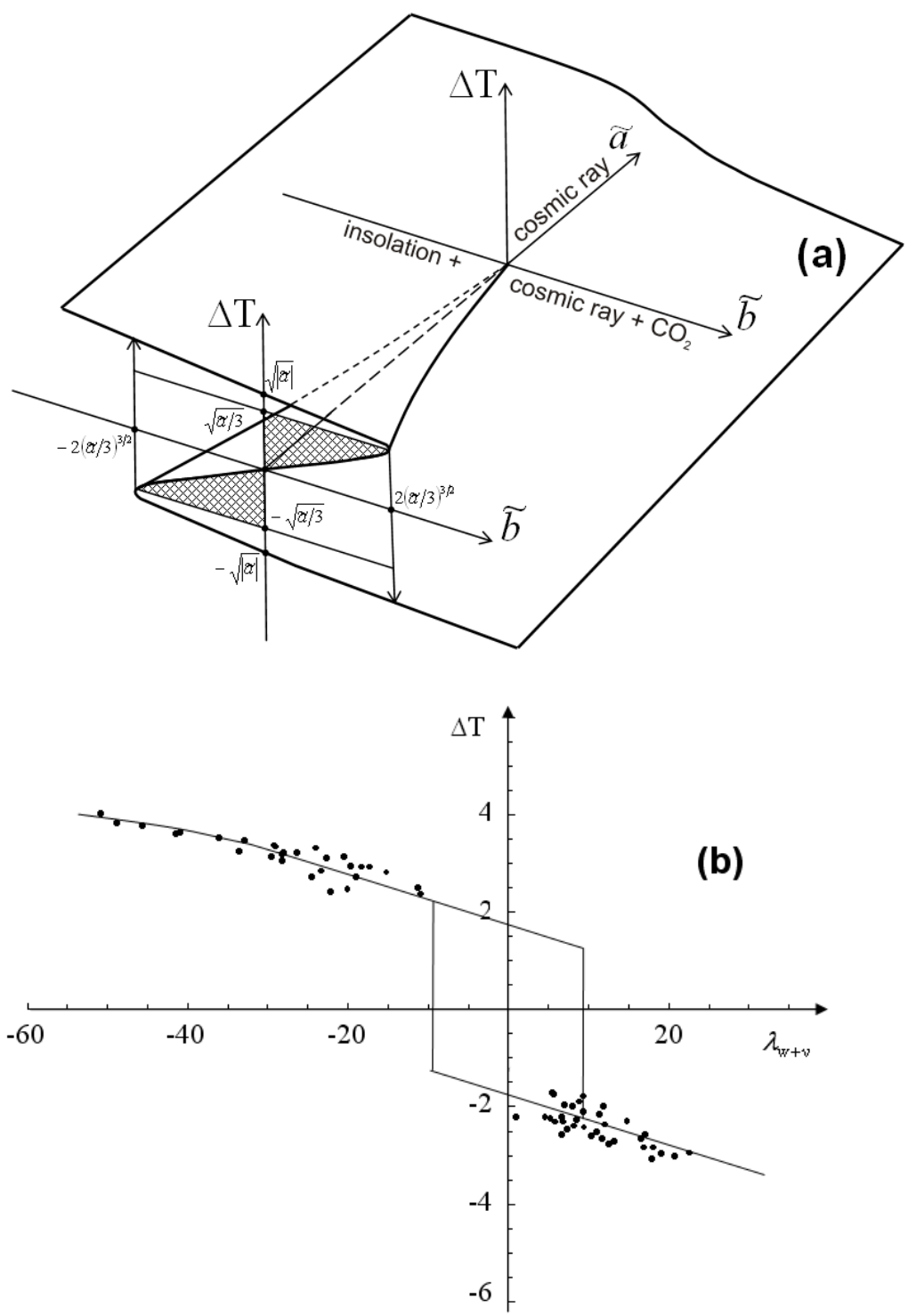

Fig.6. (a) The canonical view of variety of the fold catastrophe as a set of points $(\Delta T, \tilde{a}, \tilde{b})$ comply with Eq. (28); hatched lines are the unstable regions for solutions of Eq. (28); (b) analytical (thin curve) and calculated (•) representation of the temperature as a function of climatic sensitivity $\lambda_{w+v}$ or as function of perturbation of global freshwater or global ice volume.

$$
\frac{d}{d(\Delta T)} \Delta U^{*}(\Delta T, t)=\Delta T^{3}+\tilde{a}(t) \cdot \Delta T+\tilde{b}(t)=0
$$

Thus the general bifurcation problem contained in the arriving at a solution $\Delta \mathrm{T}(\mathrm{t})$ is reduced to the determination of the solution set of Eq. (22) for the appropriate joint trajectory $\{\tilde{a}(t), \tilde{b}(t)\}$ in the space of controlling parameters (Fig. 6a).

\section{Numerical experiment}

It is obvious that in order to solve the bifurcation equation (28) that describes extreme increments of temperature $\Delta \mathrm{T}$, it is necessary to determine the values of three climatic constants $\mathrm{a}_{\mu}, \Delta \hat{\mathfrak{R}}_{\mathrm{CO}_{2}}$, and $\Delta \hat{\mathfrak{R}}_{w+v}$ in Eqs. (25)-(26); the values of other constants, with the exception of mean temperature $\mathrm{T}_{0}$, are known.

Also, we use the experimental data of Yamasaki and Oda [17] (see Fig. 7c) as the long-term variations of relative paleointensity $M_{\oplus}^{*}(\mathrm{t})(\mathrm{Fig}$. 7c), which determine the the NRM (natural remanent magnetization) intensities normalized by ARM (anhysteretic remanent magnetization), 


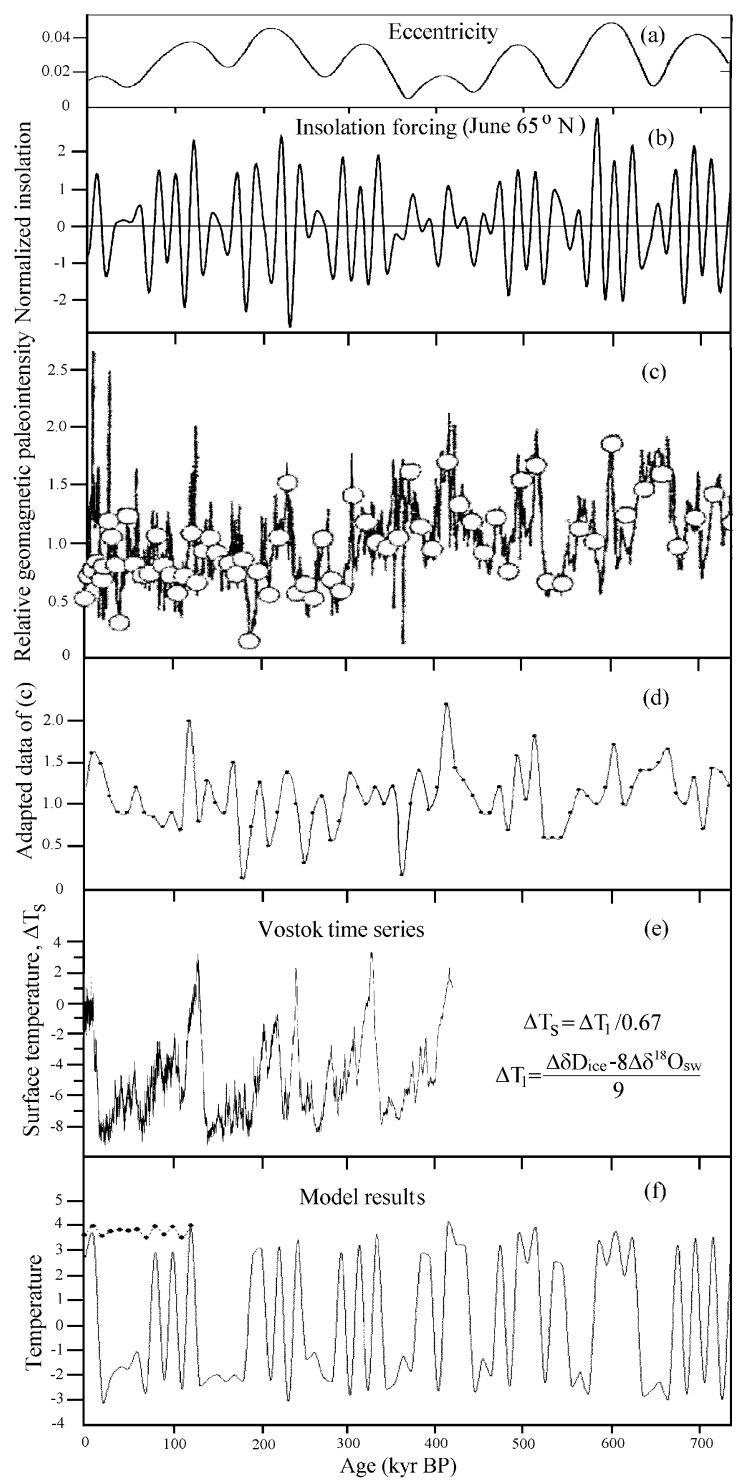

$\frac{1}{\gamma} G_{\mathrm{CO}_{2}}\left(T_{0}, t\right)=\beta T_{0} \approx 2 \cdot 1.7 \quad\left[\mathrm{~W} / \mathrm{m}^{2}\right]$

from which

$$
\Delta \hat{\mathfrak{R}} \approx 2 \frac{1.7}{\sigma_{S} T_{0}}=3.2 \cdot 10^{-4} \quad\left[W / m^{2} K\right],
$$

Fig. 7. Model of climatic response insolation and magnetic field variations of the past $730 \mathrm{kyr}$ compared with isotopic temperature data on climate of the past 420 kyr. Variations in orbital eccentricity (a) and insolation (b) at $65^{\circ} \mathrm{N}$ at the summer solstice over the past 730 kyr [Berger, 1978]. Variations of magnetic paleointensity (c) over the past $730 \mathrm{kyr}$ [Yamasaki and Oda, 2002] and adapted data (d) of magnetic paleointensity (c). Vostoc time series of isotopic temperature $\Delta \mathrm{T}_{\mathrm{S}}$ (e) at the surface [Petit et al., 1999] and result of our model calculated (f) by Eq. (28): evolution of the increment of temperature $\Delta \mathrm{T}$ relative to the average temperature $T_{0}=286.6 \mathrm{~K}$ over the past 730 kyr. Any underestimates of temperature changes $\Delta T_{S}$ (e) are defined by underestimates in the design formulae (e), where $\Delta \mathrm{T}_{1}$ is the temperature at the atmospheric (inversion) level, $\Delta \delta \mathrm{D}_{\text {ice }}$ and $\Delta \delta^{18} \mathrm{O}_{\text {sw }}$ is the globally averaged change from today's value of isotopic content of snow $\delta D_{\text {ice }}$ and seawater $\delta^{18} \mathrm{O}$ respectively. Dotted line (f) is the solution of bifurcation equation (28) under fixed magnetic field $\left(M_{\otimes}^{n}(t) \equiv 2\right)$.

Selection of the value of the mean temperature $T_{0}$ was made from the following considerations. The value of the modern climatic representative temperature is about $\mathrm{T}=288.6 \mathrm{~K}$ [28]. On the other hand, according to the data of Russian-USA-French collaboration of the Vostoc ice core [22] the mean value of the range of increment $\Delta \mathrm{T}=[2,-6]$ is less approximately on $2 \mathrm{~K}$ than the modern temperature. Therefore the value of the mean temperature $\mathrm{T}_{0}=286.6 \mathrm{~K}$ is used for further calculations.

The normalized value is $\Delta \hat{\mathfrak{R}}_{\mathrm{CO}_{2}}=\varepsilon_{\mathrm{CO}_{2}} \beta / \sigma_{\mathrm{S}}$ determined by means of known estimation for the rate of heat energy re-emitted by the carbon dioxide [29]

where the coefficient equal to 2 allows for an isotropy of radiation by the carbon dioxide, and the statistical estimation $\sigma_{\mathrm{S}} \sim\left(\mathrm{S}_{0}\right)^{1 / 2} \approx 37.1$ is used as the standard error of insolation variations.

At the same time, to determine climatic constants $a_{\mu}$ and $\Delta \hat{\mathfrak{R}}_{w+v}$ the traditional approach of adjustment with respect to experimental data. Using the Vostoc ice core data [22], one can be concluded that the sudden change of temperature at $\mathrm{t}=120 \mathrm{kyr} \mathrm{BP}$ was $\Delta \mathrm{T} \cong 4 \mathrm{~K}$. This results in the following single-valued representation of bifurcation equation (28):

$$
\Delta T^{3}-12 \Delta T-16=0 \quad \text { for } \quad t=120 k y r .
$$

Comparison of Eqs. (25), (26), and (31) implies that

$$
\begin{aligned}
& \tilde{a}_{120}=-\left.\tilde{a}_{0} a_{\mu} M_{\oplus}^{*}\right|_{t=120}=-12 \\
& \tilde{b}_{120}=-\tilde{b}_{0}\left[\left.\Delta \hat{S}\right|_{t=120}+\frac{4}{\eta_{\alpha}} \cdot \Delta \hat{\mathfrak{R}}_{C O_{2}}+\left.\frac{4}{\eta_{\alpha}} \frac{2 a_{\mu} T_{0}+b_{\mu}}{\sigma_{S}} M_{\oplus}^{*}\right|_{t=120}\right]=-16 .
\end{aligned}
$$


It should seem that the procedure for the determination of $\Delta \mathrm{T}(\mathrm{t})$ in the bifurcation problem (28) is the completely prepared. Nevertheless, calculation data on long-term variations of the (normalized) global insolation $\Delta \hat{S}$ (t), similar to that calculated by Berger [30] are unfortunately inaccessible for the authors.

We therefore make use of following fact. It is known that "ice sheets influence climate because they are among the largest topographic features on the planet, create some of the largest regional anomalies in albedo and radiation balance, and represent the largest readily exchangeable reservoir of fresh water on Earth" [31]. Also, the Northern Hemisphere ice sheets influence on the global climate change is discussed within the framework of the hypothesis on the so-called effect of Notthern's substrate. The hypothesis explains the mechanism responsible for the quasi-synchronization of the climates in the Northern and Southern Hemispheres at orbital time scales despite asynchronous insolation forcing, and lies in the assumption that "the effect of the substrate underlying the Northern Hemisphere ice sheets is to modulate ice-sheet response to insolation forcing" [31].

In view of this hypothesis as well as the fact that the long-term variations of June insolation at $65^{\circ} \mathrm{N}$ are used for the simulation of the Northern Hemisphere ice volume [32], we use these variations instead of calculation data on longterm variations of global insolation to find approximate solutions of set (32)-(33) and bifurcation equation (28). In other words, the long-term variations of June insolation at $65^{\circ} \mathrm{N}$ (Fig. 7c) are considered as first rough approximation of global insolation. Subject to this artificial approximation, the set of equations (32)-(33) yields the following solution with regard to the climatic constants $\mathrm{a}_{\mu}$ and $\mathrm{b}_{\mu}$ under $\left.M_{\oplus}^{*}\right|_{\mathrm{t}=120=2 \text { : }}$

$$
\begin{aligned}
& a_{\mu}=0.213 \\
& b_{\mu}=\frac{\eta_{\alpha} \sigma_{S}}{\left.4 M_{\otimes}^{n}\right|_{t=120}}\left[\frac{16}{\tilde{b}_{0}}-\left.\Delta \hat{S}\right|_{t=120}-\frac{4}{\eta_{\alpha}} \Delta \hat{\mathfrak{R}}_{C O_{2}}-\left.\frac{4}{\eta_{\alpha}} \frac{2 a_{\mu} T_{0}}{\sigma_{S}} M_{\oplus}^{*}\right|_{t=120}\right]=-121.820
\end{aligned}
$$

With due regard Eqs. (34) and (35), let us write down the coefficients of the bifurcation equation (28) in the final shape:

$$
\begin{aligned}
& \tilde{a}(t)=-6 M_{\oplus}^{*}(t), \\
& \tilde{b}(t)=-4.16\left[\Delta \hat{S}(t)+0.043+0.993 M_{\oplus}^{*}(t)\right] .
\end{aligned}
$$

Fig. 7f shows graphically the numerical solution of Eq. (28), which describes time evolution of $\Delta \mathrm{T}$ with respect of the mean temperature $\mathrm{T}_{0}=286.6 \mathrm{~K}$. To compare our results with experimental data, Fig. $7 \mathrm{e}$ shows the paleotemperature data from the Vostoc ice core [22].

If consider the known uncertainty of formulae used for the calculation of surface temperature $\Delta \mathrm{T}_{\mathrm{S}}$, the agreement between the theoretical (Fig. 7f) and experimental (Fig. 7e) data is quite close. The uncertainty is resulted by the impossibility to allow for the gravity correction affecting the isotope sedimentation rate in the medium [22]. On the other hand, the high uncertainty is seemingly conditioned on the actually complete neglect of the atmospheric electrical field variations initiated by the cosmic rays in low clouds. The influence of the Earth magnetic field variations on the spatial structure of water in cloud droplets and, thereafter, on the concentration of isotopes of hydrogen and oxygen (in particular, $\delta \mathrm{D}$ and $\delta^{18} \mathrm{O}$ ) is also neglected within the modern version of Polling clathrate model [33].

It is noteworthy that the solution of the bifurcation equation (28) for the settled magnetic field $\left(M_{\oplus}^{n}(t) \equiv 2\right)$, i.e. for the premeditated neglect of the GCR-clouds effect influence, results in the output (dotted line in Fig. 7f) that not represents the real variations of the experimental data from the Vostoc ice core. From mathematical view, this output comes out from the fact that (i) in this case $\tilde{a}(t)=12$ always in Eq. (36), and (ii) the term $(0.043+0.993 \cdot 2=2.029)$ in Eq. (37) is practically always larger than any negative variation of insolation. This causes the steady positive solution of Eq. (28), which is moreover larger than 3, i.e. $\Delta \mathrm{T} \geq 3 \mathrm{~K}$ (see Fig. 7f).

Thus the high goodness of fit between the experimental (Fig. 7e) and theoretical (Fig. 7e) data is a validity indicator of main assumption used in our model (see comments to Eq. (5)); we assume that the temperature of ECS is caused both the variations of insolation and variations of GCR intensity (or, equivalently, variations of Earth magnetic field).

\section{Discussion and conclusions}

To analyze a behaviour of complex climatic system, it is convenient to utilize the so-called climatic sensitivity, e.g. in the sense of definition proposed by Wigley and Raper [34]. In our case, the climatic sensitivity, $\lambda_{w+v}$, defines a rate of change of total water mass (vapour and liquid) in the atmosphere relative to the temperature increment:

$$
\begin{aligned}
& \lambda_{w+v}=\frac{1}{\Delta t} \cdot \frac{d}{d(\Delta T)} \Delta G_{w+v}(\Delta T, t)==\frac{1}{\Delta t}\left(\tilde{a}_{0} \Delta T+\tilde{b}_{0} \frac{4}{\eta_{\alpha}} \frac{2 a_{\mu} T_{0}+b_{\mu}}{\sigma_{S}}\right) M_{\oplus}^{*}(t) \equiv \\
& \equiv \frac{1}{\Delta t} \frac{\tilde{a}_{0}}{b_{w v}}\left[\left(a_{w v}+b_{w v} \Delta T\right)-a_{w v}+\tilde{b}_{0} \frac{b_{w v}}{\tilde{a}_{0}} \frac{2 a_{\mu} T_{0}+b_{\mu}}{\sigma_{S}}\right] M_{\oplus}^{*} \sim \frac{\Delta m_{w+v}}{\Delta t},
\end{aligned}
$$


where $\Delta \mathrm{m}_{\mathrm{w}+\mathrm{v}}=\langle\rho\rangle\left(\mathrm{a}_{\mathrm{wv}}+\mathrm{b}_{\mathrm{wv}} \Delta \mathrm{T}\right) M_{\oplus}^{*},\langle\rho\rangle-$ average mixture density water mass (vapour and liquid) in the atmosphere, $\Delta \mathrm{t}$ $=10 \mathrm{kyr}-$ time scale resolution (Fig. $7 \mathrm{f}$ ).

The physical meaning of this value is clearly shown by Figs. $6 \mathrm{~b}$ and 8 , from which the climatic sensitivity is an adequate valuation of temperature bistability intrinsical to the "potential" of fold catastrophe (27). Also, the decrease (increase) of increment rate of total water mass (vapour and liquid) in the atmosphere $\left(\lambda_{\mathrm{w}+\mathrm{v}}\right)$ predetermines, by virtue of conservation law, the same by value increase (decrease) of increment rate of total fresh water mass $\left(\Delta \mathrm{F}_{\text {fwf }}\right)$ in the ECS. It is obvious from the latter that there is an interrelation between the global temperature of ECS and rate of change of fresh water mass. The variations of fresh water mass are in turn provided by the global ocean circulation [35]. Moreover, the above interrelation can also display the temperature instability in the form of so-called hysteresis loop analogous that shown in Fig. $6 b$.

Thus, along with the parameter $\lambda_{\mathrm{w}+\mathrm{v}}$, the parameter $\Delta \mathrm{F}_{\mathrm{fwf}}$ defining the rate of change of fresh water mass relative to the global temperature is also climate-sensitive parameter. Generally speaking, it can be supposed that all physical, chemical, and biological parameters displaying the temperature instability in the form of so-called hysteresis loop are members of variable climate-sensitive parameters, but, in general case, at the different geological time-scale. It is in turn obvious that the climate-sensitive parameters attached to particular geological time-scale must be linearly connected each other. This statement can be easily showed (Fig. 8) on the example of series of linearly-connected parameters:

$$
-\lambda_{w+v} \sim \Delta F_{f w f} \sim I_{V}
$$

where $\mathrm{I}_{\mathrm{V}}$ is the global ice volume, $\mathrm{k}$ is the coefficient with dimension [c.K]. Moreover, the evident correlation (see Fig. 8 ) between the known time series of seawater $\delta^{18} \mathrm{O}$ (ice volume proxy) [36, 37] and analogous time series of climatic sensitivity calculated via Eq. (38) indicates both the existence of direct linear relationship between these parameters and an forecasting ability of the discussed bifurcation model with respect of Earth climate.

On the other hand, it is known that the temperature instability of global climate like other analogous instabilities relating to bistable systems can causes the regime of stochastic resonance under certain conditions [38]. For example, using an oceanatmosphere climate model Ganopolski and Rahmstorf [39] ascertained that the stochastic resonance could be an important mechanism for millennial-scale climate variability during glacial times. Also, the modeled warm events agree in many respects with the properties of the Dansgaard-Oeschger (DO) events recorded in Greenland ice and other climate archives [39]. This important outcome conforms very well with experimental data. It is noteworthy that this outcome is easily described by the proposed bifurcation model of Earth climate.

So the amplification by noise weak periodic signal acting on a multistable system is known as stochastic resonance (SR). In this sense SR is one of the most interesting noise-induced phenomenon that arises from the interplay between deterministic and random dynamics in a nonlinear system. В упрощенном виде the mechanism of SR can be explained in terms the motion of an Brownian particle in a symmetric double-well potential, subjected to deterministic periodic forcing as well as white noise.

In our case, the Earth climate system lying in asymmetric double-well potential (24) can be considered as Brownian particle. "Glacial" time scale, on which an appearance of modeled warm

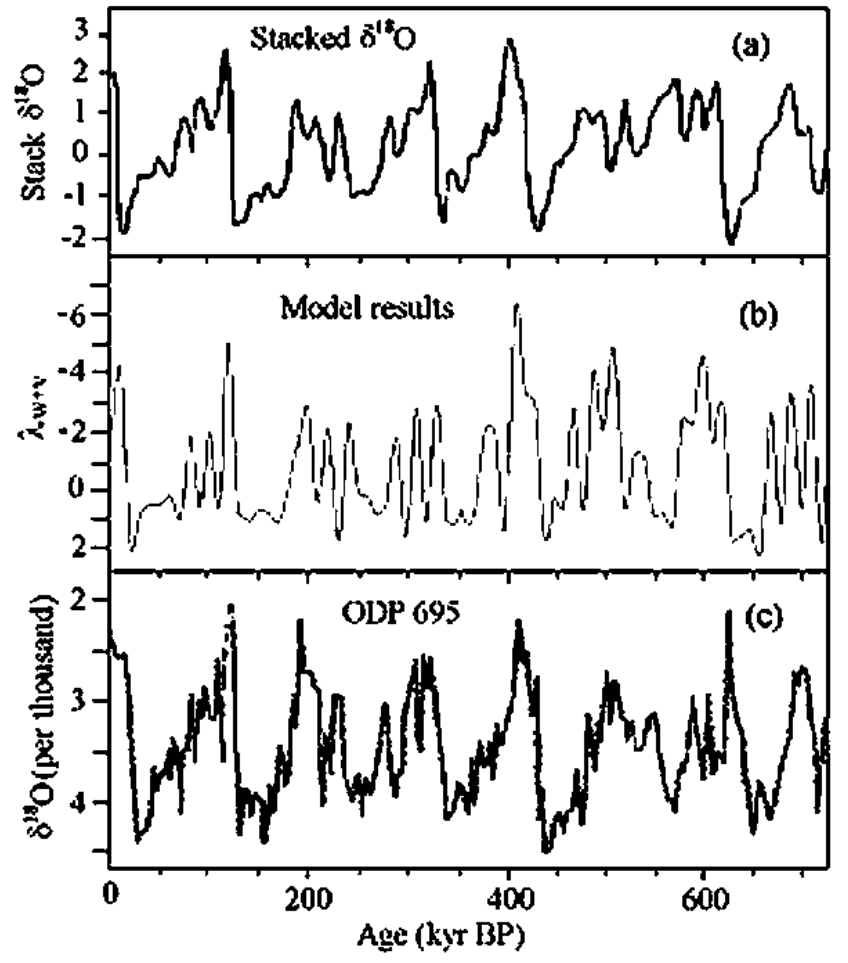

Fig. 8. Comparison of time series of seawater $\delta^{18} \mathrm{O}$ (ice volume proxy) from (a) Bassinot et al. [36] and (c) Tidemann et al. [37] with time series (b) of climatic sensitivity $\lambda_{w+v}$ (ice volume proxy) calculated by Eq. (38). 
(or DO) events can be expected, is in the 20-75 kyr interval (see Fig. 7c). On the one hand, this theoretical interval coincides with the analogous experimental interval for the actual DO events obtained by means of high-resolution stable oxygen isotopic NGRIP-record of Northern Hemisphere climate extending into the last interglacial period (105 kyr BP) [40]. On the other hand, in view of the fact that the temperature at this interval in our case is approximately constant, i.e. $\Delta \mathrm{T} \approx \mathrm{const}$, the coefficients of asymmetric double-well potential (24) are also constant at this interval. In other words, Eq. (24) can be rewritten as follows

$$
\Delta U(\Delta T, t)=\frac{1}{4} \Delta T^{4}-\frac{1}{2} \tilde{a}_{D O} \Delta T^{2}-\tilde{b}_{D O} \Delta T, \text { where } \tilde{a}_{D O}, \tilde{b}_{D O}>0
$$

It is noteworthy that the averaging of isotope data in the NGRIP-record is carried out subject to the high time-scale resolution $(\Delta \mathrm{t}=50 \mathrm{yr})$. This implies that owing to the known spectrum of temperature variations in the Northern Atlantic [16] the oscillation spectrum of stochastic climate, which is characterized by the mixture of white noise and 1/f- noise, prevails in the ECS at this time interval. Taking into account that the SR in a bistable potential is fully characterized as a synchronization effect of the hopping mechanism induced by the external periodic bias, e.g. weak harmonic signal Asin $\omega t$, for the overdamped system the dependence $\Delta \mathrm{T}(\mathrm{t})$ is to be found from

$$
\dot{x}=-\partial\left(\Delta U^{*}\right)_{x}(x, t)+A \sin \omega t+\zeta(t), \quad x(t) \equiv \Delta T(t),
$$

or

$$
\dot{x}=\tilde{a}_{D O} x-x^{3}+\tilde{b}_{D O}+A \sin \omega t+\zeta(t),
$$

where $\dot{x}$ is the temperature "friction" against the walls of potential well, $\zeta(\mathrm{t})=\xi(\mathrm{t})+\eta(\mathrm{t})$ is the additive mixture of white noise and 1/f-noise respectively.

At large noise, the term $-\tilde{b}_{D O} \mathrm{x}$ causing the asymmetry in (39) и (40) can be neglected. In view of this fact and other fact that the white noise only is applied in the computational experiment modeling the abrupt glacial climate changes due to SR [39], Eq. (42) can be rewritten as

$$
\dot{x}=\tilde{a}_{D O} x-x^{3}+A \sin \omega t+\xi(t), \quad x(t) \equiv \Delta T(t),
$$

where the amplitude of weak external periodic signal is confined to weak-forcing limit $\operatorname{Ax}_{\mathrm{m}}<<\Delta\left(\Delta \mathrm{U}^{*}(\mathrm{x}, \mathrm{t})\right)=\Delta \mathrm{U}^{*}(0)-$ $\Delta \mathrm{U}^{*}\left(\mathrm{x}_{\mathrm{m}}\right)$. Here, $\pm \mathrm{x}_{\mathrm{m}}=\left(\tilde{a}_{D O}\right)^{1 / 2}$ denote the potential minima and $\Delta\left(\Delta \mathrm{U}^{*}(\mathrm{x}, \mathrm{t})\right)=\tilde{a}_{D O}^{2} / 4$ is the potential barrier, $\xi(\mathrm{t})$ is a white Gaussian zero-mean-valid noise with correlation function $\langle\xi(\mathrm{t}), \xi(0)\rangle=2 \mathrm{D} \delta(\mathrm{t})\rangle$.

In most cases, it is convenient to identify the SR effect using an experimental investigation of the residual-time statistics for the considered system in the potential well or, more precisely, using the so-called the residence-time distribution [41]

$$
\begin{aligned}
& P(t) \sim \exp \left(-W_{K} t\right), \\
& W_{K}=\frac{1}{\tau_{K}}=\frac{1}{2 \pi}\left[\left|\frac{d^{2} \Delta U^{*}}{d x^{2}}\right|_{x=0}\left|\frac{d^{2} U^{*}}{d x^{2}}\right|_{x=x_{m}}\right]^{1 / 2} \exp \left(-\frac{\left.\Delta U^{*}\right|_{x=0}-\left.\Delta U^{*}\right|_{x=x_{m}}+x_{m} A \sin \omega t}{D}\right),
\end{aligned}
$$

where $\mathrm{W}_{\mathrm{K}}$ and $\tau_{\mathrm{K}}$ is the modified Kramer's rate и Kramer's time respectively [38]. These values define the rate and time for the transition of excited system through potential barrier under the conditions of weak periodic perturbation.

It is obvious that due to the existence of periodic component in Eq. (45) a series of peaks centered of odd multiples of the half driving period

$$
T_{n} \cong(2 n+1) \frac{\pi}{\omega}=(2 n+1) \frac{T_{\omega}}{2}, \quad n=0,1, \ldots
$$

can be observed when the function of residence-time distribution (44) making. In Eq. (46), $\omega$ and $\mathrm{T}_{\omega}$ are the frequency and period of external periodic perturbation respectively. Moreover, it follows from this the analysis that the peaks (46) appear only when the weak harmonic perturbation including; in an opposite case the monotone exponential decay of function (44) is observed. Thus the physical interpretation of Eq. (44) is obvious enough and lies in the fact that an exponential background is the part of the residence-time distribution function (44), which is produced by the changeovers caused by the noise only, whereas the peaks (46) are the reflections of synchronization between the escape mechanisms and external periodic perturbation.

Just using the ideology of optimal synchronization, when the equation of stochastic resonance in the form of Eq. (43) modeling in the framework of the bistable climate model, allows making the "experimental" function of the residence-time distribution [39]. It is also turned out that the properties of the modeled warm events, using which the residence-time distribution was obtained, are in good agreement with the actual properties of the Dansgaard-Oeschger events recorded in Greenland ice and other climate archives [39].

Finally, let us adduce the short comment for the so-called "doubling $\mathrm{CO}_{2}$ " problem. A simple analysis of structure parts in the time-dependent controlling parameter $\tilde{b}(\mathrm{t})$ denotes the fact that it contains the three independent 
components (see Eq. (26)): the variations of insolation $\Delta \hat{S}(\mathrm{t})$ and radiation forcing of total mass water (vapour and liquid) in the atmosphere $\Delta \hat{\mathfrak{R}}_{w+v} M_{\oplus}^{*}(t)$, as well as the climatic constant $\Delta \hat{\mathfrak{R}}_{\mathrm{CO}_{2}}$ defining the degree of change for the differential radiation forcing of $\mathrm{CO}_{2}$. Also, using Eq. (26) it is easily shown that for any $\mathrm{t}=0 \div 730$ kyr the inequality

$$
\Delta \hat{\mathfrak{R}}_{C O_{2}}<<\left|\frac{\eta_{\alpha}}{4} \Delta \hat{S}(t)+\Delta \hat{\mathfrak{R}}_{w+v} M_{\oplus}^{*}(t)\right|, \quad \text { for } \quad \forall t \in[0,730 \mathrm{kyr}]
$$

applies practically always (with the rare exception; see Fig. 7f).

Inequality (47) implies the fact that considerable influence of anthropogenic perturbation is only possible when the differential rate of heat energy re-emitted by the carbon dioxide, $\left(\Delta \hat{\mathfrak{R}}_{\mathrm{CO}_{2}}\right)_{\text {perturb }}$, is appreciably increased in comparison with the present-day $\Delta \hat{\mathfrak{R}}_{\mathrm{CO}_{2}}$. For example, using evident modification of Eq. (37) it is easily shown by means of computational experiment on the basis of Eq. (28) and/or (38) that at the interval $\mathrm{t}=0 \div 120 \mathrm{kyr}$ the threshold anthropogenic thermal effect occurs under the 15-time decrease of the present-day $\Delta \hat{\mathfrak{R}}_{\mathrm{CO}_{2}}$. In other words, the socalled anthropogenic "doubling $\mathrm{CO}_{2}$ " problem is practically absent in the framework of the discussed bifurcation model of Earth climate.

It can be concluded from the above mentioned that the most important, in our opinion, statement of presented model is the fact that the Earth climate, on the one hand, is completely defined by the two controlling parameters insolation and galactic cosmic rays - and, in the other hand, is quite predictable on the millennial time scales if only theoretical or experimental values on long-term variations of relative paleointensity $M_{\oplus}^{*}(\mathrm{t})$ are present.

\section{References}

1. Marsh N.D. and Svenmark H. Low Cloud Properties Influenced by Cosmic Rays / 2000, Phys. Rev. Lett., V.15. P.5004-5007.

2. Stozhkov Y.I. The role of cosmic rays in the atmospheric processes / 2003, J. of Physics G: Nuclear and Particle Physics. V.29. P. 913-923.

3. Shaviv N.J. Cosmic Ray Diffusion from the Galactic Spiral Arms, Iron Meteorites and a Possible Climate Connection / 2002, Phys. Rev. Lett., V.89. 051102; The spiral structure of the Milky Way, cosmic rays, and ice age epochs on Earth / New Astronomy 8 (2003) 39-77.

4. Lyon J.G. The Solar Wind-Magnetosphere-Ionosphere System / 2000. Nature. V.288. P.1987-1991.

5. Rind D. The Sun's Role in Climate Variations / 2002. Nature. V.296. P.673-677.

6. Ney E.R. Cosmic Radiation and Weather / 1959. Nature. V.183. P.451-452.

7. Bazilevskaya G.A. Solar cosmic rays in the near Earth space and the atmosphere / 2004. Advanced in Space Research.

8. Svensmark H. and Friis-Christensen E. 1997. J. Atmos. Sol.-Terr. Phys. V.59. P.1225-1232.

9. Svensmark H. Influence of Cosmic Rays on Earth's Climate / 1998, Phys. Rev. Lett., V.81. P.5027-5030.

10. Twomey S. 1977, J. Atmos. Sci., V.34. P.1149.

11. Kaufman Y.J., Fraser R.S. The effect of Smoke Particles on Clouds and Climate Forcing / 1997, Science. V.277. P. 1636.

12. Breon F.-M., Tanre D., Generoso S. Aerosol Effects on Cloud Droplet Size Monitored from Satellite / 2002, Science. V.295. P. 834-838.

13. Kaufman Y.J., Tanre D., Boucher O. A satellite view of aerosols in the climate system / 2002, Nature. V.419. P. 215-223.

14. Carslow K.S., Harrison R.G., Kirkby J. Cosmic Rays, Clouds, and Climate / 2002, Science. V.298. P. $1732-1737$.

15. Kulmala M. How Particles Nucleate and Grow /2003, Science. V.302. P. 1000-1001.

16. Kutzbach J.E., Bryson R.A. Variance spectrum of Holocene climatic fluctuations in North Atlantic Sector / 1974. J. Atm. Sci. V.31. P.1959-1963.

17. Yamasaki T. and Oda H. Orbital Influence on Earth's Magnetic field: 100,000-Year Periodicity in Inclination / 2002, Science. V.295. P. 2435-2438.

18. Malkus W.V.R. Precession of the Earth as the cause geomagnetism / 1968, Science. V.160. P. 259-264.

19. Consolini G. and De Michelis P. Stochastic resonance in Geomagnetic Polarity Reversals / 2003, Phys. Rev. Lett., V.90. 058501-1.

20. Twomey S., in Atmospheric Aerosols (Elsiever Science, New York, 1977), P. 278-290.

21. Rosenfeld D. Suppression of Rain and Snow by Urban and Industrial Air Pollution / 2000, Science. V.287. P. 17931796.

22. Petit J.R., Jousel J., Raynaud D. et al. Climate and atmospheric history of the past 420,000 years from the Vostoc ice core, Antarctica / 1999. Nature. V.399. P.429-436.

23. Rosenfeld D., Lahav R., Khain A., Pinsky M. The role of Sea Spray in Cleansing Air Pollution over Ocean via Cloud Processes / 2002, Science. V.297. P.1667-1670.

24. Baker M.B. and Charlson R.J. Bistability of CCN concentrations and thermodynamics in the cloud-topped boundary layer / 1990, Nature. V.345. P.142-145.

25. Albrecht B. Aerosol, Cloud Microphysics, and Fractional Cloudiness / 1989, Science. V.245. P. 1227-1230. 
26. Kirkby J., Mangini A., Muller R.A. The glacial cycles and cosmic rays / arXiv: physics/0407005 v1.

27. Gilmore R. Catastrophe Theory for scientists and engineers, Wiley-Interscience Publication, John Wiley\&Sons, New York - Chichester - Brisbane - Toronto, 1985.

28. Ghil, M. Energy-balance models: An introduction, in Climate Variations and Variability, Facts and Theories (D.Reidel, Dodrecht-Boston-London, 1980).

29. Wigley T.M.L. The Climate Change Commitment / 2005, Science. V.307. P. 1766-1769.

30. Berger A. Long-term variation of daily insolation and Quaternaty climatic change / 1978. J. Atmos. Sci. V.35. P.2362-2367.

31. Clark P.U., Alley R.B., Pollard D. Northern Hemisphere Ice-Sheet Influences on Global Climate Change / 1999, Science. V.286. P. 1104-1111.

32. Berger A. and Loutre M.F. An Exceptionally Long Interglacial Ahead ? / 2002, Science. V.297. P. 1287-1288.

33. Vysotskii V.I., Smirnov I.V., Kornilova A.A. Introduction to the Biophysics of Activated Water, Universal Publishiers, Roca Raton, Florida, 2005.

34. Wigley T.M.L. and Raper S.G.B. Natural variability of the climate system and detection of the greenhouse effects / 1990, Nature. V.344. P.324-327.

35. Wunsch C. What is the Thermohaline Circulation / 2002, Science. V.298. P. 1179-1180.

36. Bassinot F.C., Labeyrie L.D., Vincent E. et al. The astronomical theory of climate and the age of the BrunhesMatuyama magnetic reversal / 1994, Earth Planet. Sci. Lett. V.126. P.91-108.

37. Tidemann R., Sarnthein M., Shackleton N.J. Astronomic timescale for the Pliocene Atlantic $\delta^{18} \mathrm{O}$ and dust flux records of Ocean Drilling Program site 659 / 1994, Paleoceanography, V.9. P.619-638.

38. Moss F., in Some Problems in Statistical Physics (Philadelfia: SIAM, 1994) p.205-253.

39. Ganopolski A. and Rahmstorf S. Abrupt Glacial Climate Change due to Stochastic Resonance / 2002, Phys. Rev. Lett., V.88. 038501-1.

40. Anderson K.K., Azuma N., Barnola J.-M. et al. High-resolution record of Northern Hemisphere climate extending into the last interglacial period / 2004, Nature. V.431. P.147-151.

41. Gammatoni L., Marchesoni F., Santucci S. Stochastic Resonance as a Bona Fide Resonance / 1995, Phys. Rev. Lett., V.74. P.1052-1055. 